\title{
Baeyer-Villiger oxidation under Payne epoxidation conditions
}

\author{
Tyne Bradley, Andrei Dragan, and Nicholas Tomkinson*
}

WestCHEM, Department of Pure and Applied Chemistry, Thomas Graham Building, University of Strathclyde, 295 Cathedral Street, Glasgow, G1 1XL, U.K.

Absract: A novel method for the Baeyer-Villiger oxidation of ketones has been developed and optimized. The transformation involves a transition metal-free activation of hydrogen peroxide under Payne epoxidation conditions. Reaction of a ketone with hydrogen peroxide in the presence of a nitrile under mildly basic reaction conditions leads to the corresponding ester. The transformation has been successfully applied to a range of ketones in moderate to excellent yields (30 to $91 \%$ ) and good to excellent regioselectivities (7:1 to 20:1).

\section{Introduction}

Since its discovery in $1899,{ }^{1}$ the Baeyer-Villiger (BV) oxidation has developed to become a stalwart transformation in synthesis. ${ }^{2,3}$ Central to the reaction is a source of peroxide, with meta-chloroperoxybenzoic acid ( $m \mathrm{CPBA})$ being a common commercial oxidant. ${ }^{4} \mathrm{~A}$ more convenient oxidant is hydrogen peroxide, due to its low cost, high oxygen content, ease of handling and the generation of water as a byproduct. Hydrogen peroxide is available either as an aqueous solution or in anhydrous form as a urea complex, ${ }^{5,6}$ or a sodium percarbonate salt, ${ }^{7-9}$ and each of these have found broad application in synthesis.

For the BV reaction to proceed with hydrogen peroxide as the oxidant, the peroxide requires activation. ${ }^{3}$ Many activation methods involve the use of abundant Sn catalysts, the most efficient being Sn-zeolites. ${ }^{10-17}$ Other metals used for the activation of hydrogen peroxide in the $\mathrm{BV}$ oxidation include $\mathrm{Se},{ }^{18} \mathrm{Re},{ }^{19} \mathrm{Li}$ and $\mathrm{Ca}$ borates, ${ }^{20} \mathrm{Pt},{ }^{21-23} \mathrm{Pd},{ }^{24} \mathrm{Si}$ polyoxometalates, ${ }^{25}$ and $\mathrm{Zr} .{ }^{26} \mathrm{Metal}-\mathrm{free}$ methods also exist and make use of enzymes, ${ }^{27}$ ionic liquids, ${ }^{28}$ carboxylic acids,${ }^{29}$ phosphoric acids, ${ }^{30,31}$ and bisflavins ${ }^{32}$ to activate the peroxide. Of these methods, a few have been shown to be effective for enantioselective BV oxidations, which include metals such as Pt, Pd or $\mathrm{Zr}$ with chiral ligands, ${ }^{21,24,26}$ pro-chiral carboxylic acids, ${ }^{29}$ phosphoric acids, ${ }^{30,31}$ and bisflavins. ${ }^{32}$ While there are a variety of methods of activating hydrogen peroxide, it is still desirable to develop methods to broaden the transformation, specifically by defining new pathways to potential asymmetric oxidations.

A simple method using nitriles to activate hydrogen peroxide under mildly basic reaction conditions was introduced by Payne in 1961 which resulted in an effective process for the epoxidation of alkenes (Scheme 1; Conditions A) ${ }^{33,34}$ Reaction of aqueous hydrogen peroxide (50\%) with alkene 1 in the presence of benzonitrile at pH 9.5-10 gave the epoxide $\mathbf{2}$ in $70 \%$ isolated yield. This use of nitriles for the activation of hydrogen peroxide represents a convenient and simple reaction which has since been developed to provide an effective and reliable method for alkene oxidation, ${ }^{35-39}$ which has been extended to asymmetric epoxidations, ${ }^{40,41}$ and the formation of chiral oxaziridines. $^{42,43}$

Payne proposed the epoxidation proceeded via formation of a highly reactive peroxy imidic acid intermediate $\mathbf{3}$. Whilst there has been overwhelming acceptance of this suggestion, peroxy imidic acids are highly reactive and have not been isolated due to decomposition via Radziszewski oxidation leading to the corresponding amide. ${ }^{44}$ A study by Vacque et al. using attenuated total reflectance (ATR) coupled with Fourier transform infrared (FTIR) and FT Raman spectroscopy provided important experimental evidence toward the existence of such a peroxy imidic acid intermediate. ${ }^{45}$

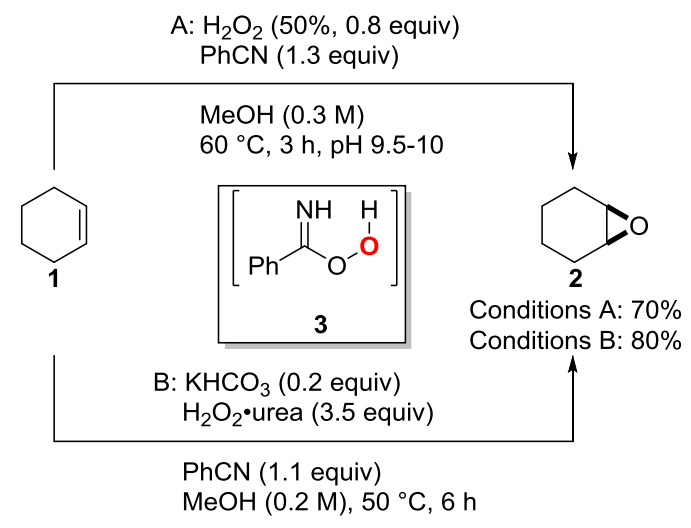

Scheme 1. Payne epoxidation.

A recent report by $\mathrm{Ji}$ described the discovery of convenient and novel conditions for the Payne epoxidation of alkenes (Scheme 1; Conditions B). ${ }^{46}$ Treatment of an alkene (e.g. 1) with an excess of hydrogen peroxide urea complex in the presence of benzonitrile and potassium bicarbonate at $50{ }^{\circ} \mathrm{C}$ gave the epoxide 2 in $80 \%$ isolated yield after purification by column chromatography. Of particular note within this transformation was the practicality of the procedure using a convenient source of peroxide in the presence of a catalytic amount of base and the mild conditions under which the reaction proceeded. 
Whilst a substantial amount of work has been devoted to the development of the Payne epoxidation, relatively little work has been undertaken in exploiting the oxidation potential of the peroxy imidic acid intermediates (e.g. 3) in alternative oxidation processes. This is specifically the case for the BV reaction where the isolated work of Ruiz investigated specialized heterogeneous magnesium, ${ }^{47}$ or magnesium/aluminum hydrotalcite supports as catalysts for the transformation. ${ }^{48,49}$ This work showed the reaction of a ketone $(e . g .4)$ in benzonitrile solvent with aqueous hydrogen peroxide, a heterogeneous catalyst and the surfactant DBS could lead to the corresponding ester or lactone (e.g. 5) (Scheme 2). Whilst effective, the need to prepare the catalyst through specific protocols and the use of benzonitrile as the solvent has limited the uptake of this method by the synthetic community. Within this current work we develop conditions for the activation of hydrogen peroxide with a nitrile which can be applied to the BV oxidation providing a convenient and reliable method for the preparation of both esters and lactones from the corresponding ketone.

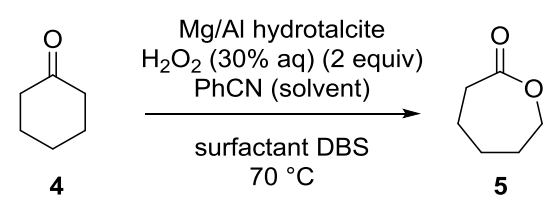

Scheme 2. Heterogeneous Baeyer-Villiger oxidation in benzonitrile.

\section{Results and discussion}

\subsection{Initial result}

Our investigations began by the reaction of cyclohexanone $\mathbf{4}$ with urea hydrogen peroxide complex under conditions reported by Ji for alkene epoxidation (Scheme 3). ${ }^{46}$ We were delighted to discover that $\varepsilon$-caprolactone 5 was formed in $38 \%$ conversion by ${ }^{1} \mathrm{H}$ NMR spectroscopy after 48 hours. Repeating the reaction in the absence of $\mathrm{KHCO}_{3}$, or $\mathrm{H}_{2} \mathrm{O}_{2}$. urea, or PhCN resulted in no product 5 being formed showing that all components added were necessary to bring about the transformation.

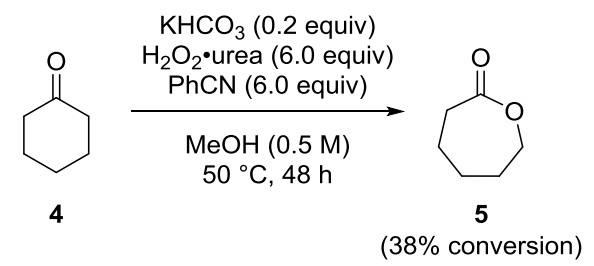

Scheme 3. Baeyer-Villiger oxidation using benzonitrile for peroxide activation.

\subsection{Optimization of the Baeyer-Villiger reaction}

Based upon the excellent result outlined in Scheme 3, we elected to optimize the reaction conditions for this BV transformation. Table 1 provides a selection of the data obtained during this optimization process examining the concentration, base, peroxide source, solvent, reaction stoichiometry and temperature.

To accelerate the optimization process initial experiments were conducted in methanol- $d_{4}$ monitoring by ${ }^{1} \mathrm{H}$ NMR spectroscopy and using 1,4-dinitrobenzene as an internal standard. Both increasing (entry 2;30\%) and decreasing (entry 3; 33\%) the concentration of the reaction resulted in a lower conversion under the conditions examined. Reducing the temperature significantly slowed down the reaction (14\%; entry 5), whilst performing the reaction at reflux had negligible effect on the conversion (44\%; entry 6). Altering the peroxide source to $\mathrm{H}_{2} \mathrm{O}_{2}(30 \% \mathrm{v} / \mathrm{v}$ aqueous solution) resulted in a considerably worse transformation (31\%; entry 9) suggesting that the urea hydrogen peroxide complex was an integral part of the process. Reducing the stoichiometry of both the nitrile (10\%; entry 11) and the peroxide (25\%; entry 7) also proved detrimental to the transformation. Increasing the amount of base resulted in an increased consumption of starting material, but this was accompanied by decomposition of the product as confirmed against the internal standard (entries 4 and 14).

Careful examination of the crude reaction mixtures showed a small amount of the $\varepsilon$-caprolactone $\mathbf{5}$ was undergoing solvolysis with methanol to give the corresponding $\varepsilon$-hydroxy ester. We therefore considered reducing the nucleophilicity of the alcoholic solvent to shut down this unwanted side reaction and investigated the use of fluorinated alcohols as the reaction medium. Interestingly, it has been shown that fluorinated alcohols can be used to accelerate reactions involving peroxides. ${ }^{50,51}$ It has also been shown that fluorinated alcohols have the ability to activate hydrogen peroxide, ${ }^{52}$ which has been specifically exploited in oxidation reactions. Examination of hexafluoroisopropanol (HFIP) as an alternative reaction solvent resulted in an equivalent conversion of cyclohexanone $\mathbf{4}$ to the ester $\mathbf{5}$ (42\%; entry 12). Importantly, within this transformation no reaction of $\mathbf{5}$ with the solvent was observed by ${ }^{1} \mathrm{H}$ NMR spectroscopy. Changing the solvent to trifluoroethanol (TFE) showed a significantly improved conversion (76\%; entry 13) which was confirmed by isolation of the product after aqueous workup and column chromatography (70\%). Once again, control experiments in the absence of benzonitrile and/or base $\left(\mathrm{KHCO}_{3}\right)$ confirmed that both were necessary for the reaction to proceed efficiently in TFE.

Table 1. Optimization of the Baeyer-Villiger reaction produced via Scheme $3 .^{a}$

\begin{tabular}{ccccccc}
\hline Entry & $\begin{array}{c}\mathbf{H}_{2} \mathbf{O}_{2} \text { source } \\
\text { (equiv) }\end{array}$ & $\begin{array}{c}\mathbf{K H C O}_{3} \\
\text { (equiv) }\end{array}$ & $\begin{array}{c}\text { Nitrile } \\
\text { (equiv) }\end{array}$ & $\begin{array}{c}\text { Solvent } \\
(\mathbf{M})\end{array}$ & $\begin{array}{c}\mathbf{T} \\
\left({ }^{\circ} \mathbf{C}\right)\end{array}$ & $\begin{array}{c}\text { Conversion } \\
(\%)^{b}\end{array}$ \\
\hline 1 & $\mathrm{H}_{2} \mathrm{O}_{2} \bullet$ urea $(6.0)$ & 0.2 & $\mathrm{PhCN}(6.0)$ & $\mathrm{MeOH}(0.5)$ & 50 & 38
\end{tabular}




\begin{tabular}{|c|c|c|c|c|c|c|}
\hline 2 & $\mathrm{H}_{2} \mathrm{O}_{2} \cdot$ urea $(6.0)$ & 0.2 & $\mathrm{PhCN}(6.0)$ & $\mathrm{CD}_{3} \mathrm{OD}(1.0)$ & 50 & 30 \\
\hline 3 & $\mathrm{H}_{2} \mathrm{O}_{2} \cdot$ urea $(6.0)$ & 0.2 & $\mathrm{PhCN}(6.0)$ & $\mathrm{CD}_{3} \mathrm{OD}(0.2)$ & 50 & 33 \\
\hline 4 & $\mathrm{H}_{2} \mathrm{O}_{2} \cdot$ urea $(6.0)$ & 0.5 & $\mathrm{PhCN}(6.0)$ & $\mathrm{CD}_{3} \mathrm{OD}(0.5)$ & 50 & $60^{c}$ \\
\hline 5 & $\mathrm{H}_{2} \mathrm{O}_{2} \cdot$ urea (6.0) & 0.2 & $\mathrm{PhCN}(6.0)$ & $\mathrm{CD}_{3} \mathrm{OD}(0.5)$ & 25 & 14 \\
\hline 6 & $\mathrm{H}_{2} \mathrm{O}_{2} \cdot$ urea $(6.0)$ & 0.2 & $\mathrm{PhCN}(6.0)$ & $\mathrm{CD}_{3} \mathrm{OD}(0.5)$ & 65 & 44 \\
\hline 7 & $\mathrm{H}_{2} \mathrm{O}_{2} \cdot$ urea (3.0) & 0.2 & $\mathrm{PhCN}(6.0)$ & $\mathrm{CD}_{3} \mathrm{OD}(0.5)$ & 50 & 25 \\
\hline 8 & $\mathrm{H}_{2} \mathrm{O}_{2}$ •urea (10.0) & 0.2 & $\mathrm{PhCN}(6.0)$ & $\mathrm{CD}_{3} \mathrm{OD}(0.5)$ & 50 & 47 \\
\hline 9 & $\mathrm{H}_{2} \mathrm{O}_{2} 30 \%$ (6.0) & 0.2 & $\mathrm{PhCN}(6.0)$ & $\mathrm{CD}_{3} \mathrm{OD}(0.5)$ & 50 & 31 \\
\hline 10 & $\mathrm{H}_{2} \mathrm{O}_{2} \cdot$ urea $(6.0)$ & 0.2 & $\mathrm{PhCN}(3.0)$ & $\mathrm{CD}_{3} \mathrm{OD}(0.5)$ & 50 & 21 \\
\hline 11 & $\mathrm{H}_{2} \mathrm{O}_{2} \cdot$ urea $(6.0)$ & 0.2 & $\mathrm{PhCN}(1.1)$ & $\mathrm{CD}_{3} \mathrm{OD}(0.5)$ & 50 & 10 \\
\hline 12 & $\mathrm{H}_{2} \mathrm{O}_{2} \cdot$ urea $(6.0)$ & 0.2 & $\mathrm{PhCN}(6.0)$ & $\operatorname{HFIP}(0.5)$ & 50 & 42 \\
\hline 13 & $\mathrm{H}_{2} \mathrm{O}_{2} \cdot$ urea (6.0) & 0.2 & PhCN (6.0) & TFE (0.5) & 50 & $76(70 \%)^{d}$ \\
\hline 14 & $\mathrm{H}_{2} \mathrm{O}_{2} \bullet$ urea (6.0) & 0.5 & $\mathrm{PhCN}(6.0)$ & TFE $(0.5)$ & 50 & $95^{c}$ \\
\hline 15 & $\mathrm{H}_{2} \mathrm{O}_{2} \cdot$ urea (6.0) & 0.1 & $\mathrm{PhCN}(6.0)$ & TFE $(0.5)$ & 50 & 37 \\
\hline
\end{tabular}

${ }^{a}$ Reaction of cyclohexanone (1 mmol) for $48 \mathrm{~h}$.

${ }^{b}$ Conversion was monitored by ${ }^{1} \mathrm{H}$ NMR against an internal standard (1,4-dinitrobenzene).

${ }^{c}$ Product decomposition observed.

${ }^{d}$ Isolated yield after aqueous workup and purification by column chromatography. 
Table 2. Substrate scope for the Baeyer-Villiger oxidation.

$$
\begin{aligned}
& \mathrm{KHCO}_{3} \text { (0.2 equiv) } \\
& \mathrm{H}_{2} \mathrm{O}_{2} \text {-urea (6.0 equiv) }
\end{aligned}
$$

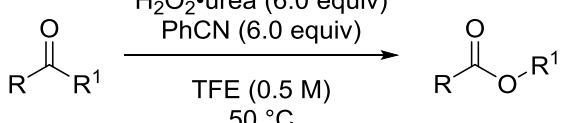

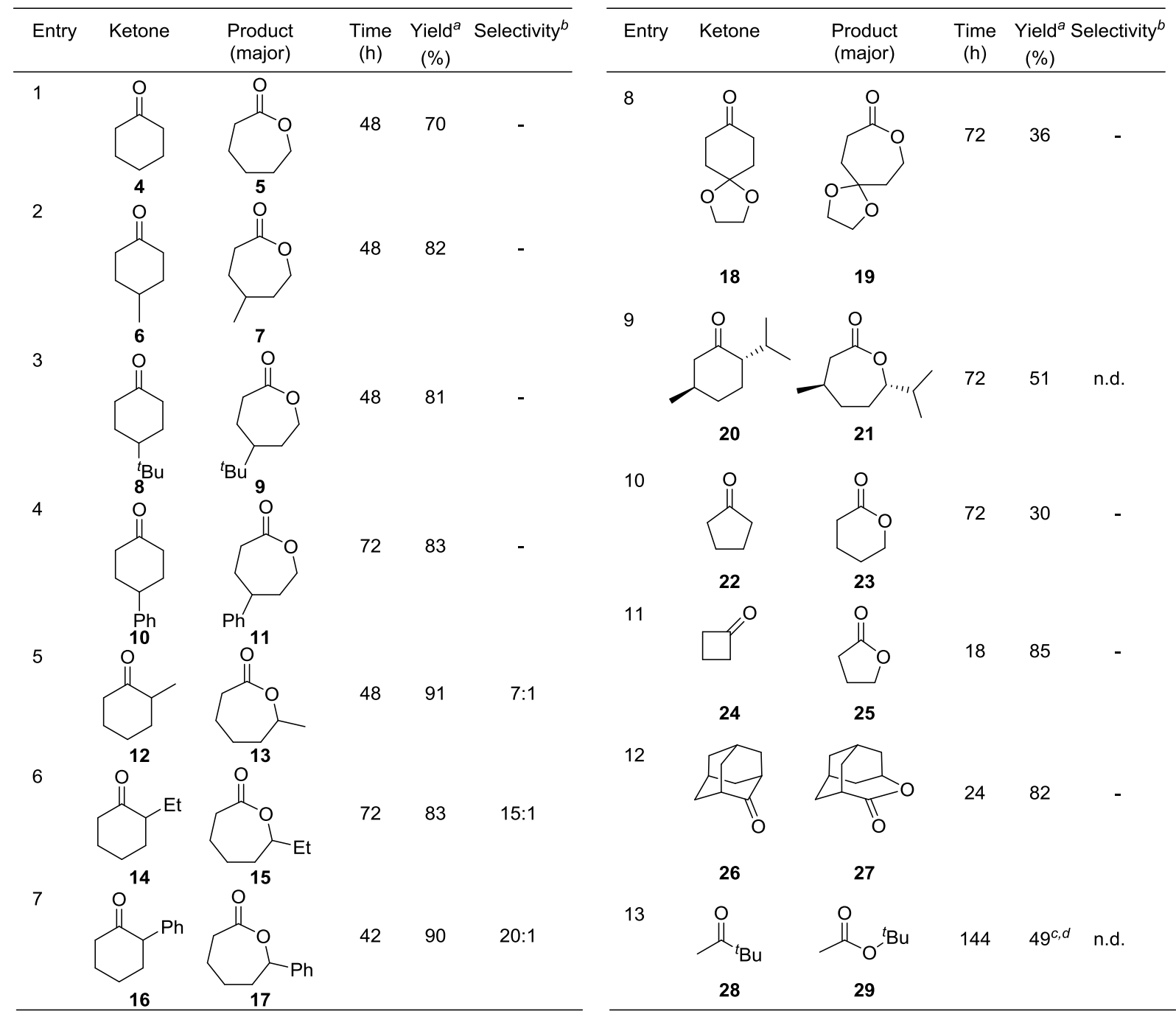

${ }^{a}$ Isolated yield.

${ }^{b}$ Ratio between regioisomeric products (major isomer shown), determined by ${ }^{1} \mathrm{H}$ NMR spectroscopy on crude reaction mixture.

${ }^{c}$ Conversion to single product monitored by ${ }^{1} \mathrm{H}$ NMR against an internal standard (1,4-dinitrobenzene).

${ }^{d}$ Average of two runs.

\subsection{Substrate scope}

Having optimized the reaction conditions we examined some of the scope and limitations of the process (Table 2). The transformation exhibited typical features of the BV oxidation in terms of relative reactivity, regioselectivity and stereoselectivity. Reactions proceeded well and in good yield for a series of cyclohexanone derivatives (entries 1-9). Control of the regioselectivity was particularly high with more substituted groups preferentially migrating (entries 5-7 and 9). Increasing the steric congestion around the reactive carbonyl slowed down the reaction, but these transformations remained clean and simply required a longer reaction time (entries 6 and 9). Retention of configuration of the migrating carbon was supported by the optical rotation of the product derived from (-)-menthone $\mathbf{2 0}$ as the substrate (entry 9). Cyclopentanone $\mathbf{2 2}$ reacted slower (30\%, $72 \mathrm{~h}$; entry 10) when compared to cyclohexanone $\mathbf{4}$ (70\%, 48 h; entry 1), whereas cyclobutanone $\mathbf{2 4}$ reacted substantially quicker, giving the expected lactone in an excellent $85 \%$ yield in just $18 \mathrm{~h}$ (entry 11). Whilst acyclic ketones were reluctant substrates, it did prove possible to react pinacolone $\mathbf{2 8}$ to give the expected product 29 after extended reaction times $(49 \%, 144 \mathrm{~h}$; entry 13) suggesting further optimization would be required to bring about efficient transformations for this class of substrate.

\section{Conclusion}

In summary, a novel BV reaction has been developed and optimized. The transformation uses urea hydrogen peroxide complex as a convenient source of peroxide which is activated by benzonitrile and a catalytic amount of base $\left(\mathrm{KHCO}_{3}\right)$ in a fluorinated alcohol to form the active peroxy imidic acid in situ. The reaction proceeds in moderate to good yields, with excellent and predictable 
regioselectivity and migration proceeding with retention of configuration. Given the marked success in developing asymmetric variants of the Payne epoxidation, we believe these results pave the way for exploring novel asymmetric BV oxidation processes.

\section{Experimental section}

\subsection{General experimental details}

Commercially available solvents and reagents were used without further purification or drying and all reactions were performed under an argon atmosphere. Flash chromatography was carried out using Merck Kieselgel $60 \mathrm{H}$ silica. Analytical thin layer chromatography was carried out using aluminum-backed plates coated with Merck Kieselgel 60 GF254 that were visualized under UV light (at $254 \mathrm{~nm}$ ) or stained using $\mathrm{KMnO}_{4}$. Nuclear magnetic resonance (NMR) spectra were recorded on a Bruker Avance III or a Bruker Avance spectrometer, operating at $400 \mathrm{MHz}\left({ }^{1} \mathrm{H}\right)$ and $101 \mathrm{MHz}\left({ }^{13} \mathrm{C}\right)$, respectively. Chemical shifts were reported in parts per million (ppm) in the scale relative to $\mathrm{CHCl}_{3}, 7.26 \mathrm{ppm}$ for ${ }^{1} \mathrm{H} \mathrm{NMR}$ and 77.16 for ${ }^{13} \mathrm{C}$ NMR. Multiplicities are abbreviated as: $\mathrm{s}$, singlet; $\mathrm{d}$, doublet; app d, apparent doublet; t, triplet; app t, apparent triplet; q, quartet; dd, doublet of doublets; app dd, apparent doublet of doublets; app dq, apparent doublet of quartets; ddd, doublet of doublets of doublets; dtd, doublet of triplets of doublets; t app d, triplet of apparent doublets; m, multiplet. Coupling constants are measured in Hertz (Hz). Low-resolution mass spectra (LRMS) were recorded on an Agilent 7890A GC system, equipped with a 30 m DB5MS column connected to a 5975C inert XL CI MSD with Triple-Axis Detector. High-resolution mass spectra (HRMS) were obtained courtesy of the EPSRC National Mass Spectrometry Facility at Swansea University, U.K. Infrared spectra were recorded on a Shimadzu IRAffinity-1 equipped with ATR (Attenuated Total Reflectance) and were reported in $\mathrm{cm}^{-1}$. Melting points were obtained on a Stuart SMP11 device. In vacuo refers to evaporation under reduced pressure using a rotary evaporator connected to a diaphragm pump, followed by the removal of trace volatiles using a high vacuum (oil) pump.

\subsection{General Baeyer-Villiger procedure (General Procedure 1)}

To a flame dried crimp top vial under argon, $\mathrm{KHCO}_{3}(80 \mathrm{mg}, 0.8 \mathrm{mmol}, 0.2$ equiv) was weighed, followed by urea hydrogen peroxide (564 mg, $24 \mathrm{mmol}, 6.0$ equiv). The ketone ( $4.0 \mathrm{mmol}, 1.0$ equiv) was added at this stage if it was a solid. The vial was then sealed and evacuated, followed by backfilling with argon (repeated three times). Afterward, the solvent ( $8.0 \mathrm{~mL})$, benzonitrile ( 2.48 $\mathrm{mL}, 24 \mathrm{mmol}, 6.0$ equiv) and, if it was a liquid, the ketone ( $4.0 \mathrm{mmol}, 1.0$ equiv), were added via syringe. The reaction was allowed to run for the specified time at $50{ }^{\circ} \mathrm{C}$, after which it was cooled down to room temperature. The pressure was released with a needle, then the vial was opened. The mixture was diluted with $\mathrm{CH}_{2} \mathrm{Cl}_{2}(40 \mathrm{~mL})$ and water $(20 \mathrm{~mL})$. The layers were separated and the resulting aqueous solution was extracted with $\mathrm{CH}_{2} \mathrm{Cl}_{2}(2 \times 40 \mathrm{~mL})$. The combined organic layers were dried $\left(\mathrm{MgSO}_{4}\right)$, concentrated in vacuo, and the crude product was subjected to flash chromatography on silica gel (5:1 petroleum ether:EtOAc) to afford the corresponding product(s).

\section{3. $\varepsilon$-Caprolactone $(5)^{53}$}

Lactone 5 was prepared according to General Procedure 1 using cyclohexanone $4(0.44 \mathrm{~mL}, 4.0 \mathrm{mmol})$. The title compound was isolated as a colourless oil $(321 \mathrm{mg}, 2.8 \mathrm{mmol}, 70 \%)$. ${ }^{1} \mathrm{H}$ NMR $\left(400 \mathrm{MHz}, \mathrm{CDCl}_{3}\right) \delta 4.28-4.17(\mathrm{~m}, 2 \mathrm{H}), 2.71-2.57(\mathrm{~m}, 2 \mathrm{H}), 1.90-1.80$ $(\mathrm{m}, 2 \mathrm{H}), 1.81-1.69(\mathrm{~m}, 4 \mathrm{H}) ;{ }^{13} \mathrm{C}$ NMR $\left(101 \mathrm{MHz}, \mathrm{CDCl}_{3}\right) \delta 176.3,69.4,34.7,29.4,29.1,23.1 ; \mathrm{LRMS}(\mathrm{CI}) \mathrm{m} / z$ 115.0 [M+H] ${ }^{+} ; \mathrm{IR}$ $(\mathrm{ATR}) / \mathrm{cm}^{-1} 2988,2901,1726$.

\subsection{5-Methyloxepan-2-one (7) ${ }^{54}$}

Lactone 7 was prepared according to General Procedure 1 using 4-methylcyclohexanone 6 ( $0.49 \mathrm{~mL}, 4.0 \mathrm{mmol})$. The title compound was isolated as a colourless oil $(421 \mathrm{mg}, 3.3 \mathrm{mmol}, 82 \%) .{ }^{1} \mathrm{H} \mathrm{NMR}\left(400 \mathrm{MHz}, \mathrm{CDCl}_{3}\right) \delta 4.28(\mathrm{ddd}, J=12.9,5.7,1.9 \mathrm{~Hz}, 1 \mathrm{H}), 4.17(\mathrm{dd}$, $J=12.7,10.4 \mathrm{~Hz}, 1 \mathrm{H}), 2.71-2.56(\mathrm{~m}, 2 \mathrm{H}), 1.98-1.83(\mathrm{~m}, 2 \mathrm{H}), 1.82-1.71(\mathrm{~m}, 1 \mathrm{H}), 1.50(\mathrm{dtd}, J=15.2,10.8,1.8 \mathrm{~Hz}, 1 \mathrm{H}), 1.33(\mathrm{dtd}, J=$ 13.9, 11.5, $2.4 \mathrm{~Hz}, 1 \mathrm{H}), 0.99(\mathrm{~d}, J=6.6 \mathrm{~Hz}, 3 \mathrm{H}) ;{ }^{13} \mathrm{C} \mathrm{NMR}\left(101 \mathrm{MHz}, \mathrm{CDCl}_{3}\right) \delta 176.3,68.3,37.4,35.4,33.4,30.9,22.3 ; \mathrm{LRMS}(\mathrm{CI})$ $m / z$. $129.0[\mathrm{M}+\mathrm{H}]^{+}$; HRMS (SIR-CI) calculated for $\mathrm{C}_{7} \mathrm{H}_{13} \mathrm{O}_{2}[\mathrm{M}+\mathrm{H}]^{+}$129.0910, found 129.0910; IR (ATR)/cm ${ }^{-1} 2988,2901,1717$.

\subsection{5-(tert-Butyl)oxepan-2-one $(\mathbf{9})^{55}$}

Lactone 9 was prepared according to General Procedure 1 using 4-tert-butylcyclohexanone 8 (617 mg, $4.0 \mathrm{mmol})$. The title compound was isolated as a white solid (554 mg, $3.3 \mathrm{mmol}, 81 \%)$. m.p. $49-50{ }^{\circ} \mathrm{C}$ (lit. $\left.49{ }^{\circ} \mathrm{C}\right) ;{ }^{55}{ }^{1} \mathrm{H} \mathrm{NMR}\left(400 \mathrm{MHz} \mathrm{CDCl}{ }_{3}\right) \delta 4.32$ (ddd, $J=12.9,5.9,1.8 \mathrm{~Hz}, 1 \mathrm{H}), 4.14(\mathrm{dd}, J=12.9,10.4 \mathrm{~Hz}, 1 \mathrm{H}), 2.69$ (ddd, $J=14.3,7.5,1.4 \mathrm{~Hz}, 1 \mathrm{H}), 2.61-2.51(\mathrm{~m}, 1 \mathrm{H}), 2.13-1.95$ $(\mathrm{m}, 2 \mathrm{H}), 1.58-1.44(\mathrm{~m}, 1 \mathrm{H}), 1.38-1.28(\mathrm{~m}, 2 \mathrm{H}), 0.88(\mathrm{~s}, 9 \mathrm{H}) ;{ }^{13} \mathrm{C}$ NMR $\left(101 \mathrm{MHz}, \mathrm{CDCl}_{3}\right) \delta 176.5,68.8,50.9,33.6,33.1,30.5,27.6$, 23.9; LRMS (CI) $m / z$ 171.1 [M+H] $]^{+}$; HRMS (FTMS-NSI) calculated for $\mathrm{C}_{10} \mathrm{H}_{19} \mathrm{O}_{2}[\mathrm{M}+\mathrm{H}]^{+} 171.1380$, found 171.1377; IR (ATR)/cm ${ }^{-1}$ 2987, 2961, 1717.

\subsection{5-Phenyloxepan-2-one $(11)^{56}$}

Lactone 11 was prepared according to General Procedure 1 using 4-phenylcyclohexanone 10 (697 mg, $4.0 \mathrm{mmol})$. The title compound was isolated as a white solid (629 mg, $3.3 \mathrm{mmol}, 83 \%)$. m.p. $95-96{ }^{\circ} \mathrm{C}\left(\right.$ lit. $\left.100-101{ }^{\circ} \mathrm{C}\right) ;{ }^{56}{ }^{1} \mathrm{H} \mathrm{NMR}\left(400 \mathrm{MHz}, \mathrm{CDCl}{ }_{3}\right) \delta$ 7.36-7.29 (m, 2H), 7.26-7.21 (m, 1H), 7.21-7.16 (m, 2H), 4.40 (ddd, $J=13.0,5.3,2.3 \mathrm{~Hz}, 1 \mathrm{H}), 4.32(\mathrm{ddd}, J=13.0,10.0,1.0 \mathrm{~Hz}, 1 \mathrm{H})$, $2.90-2.71(\mathrm{~m}, 3 \mathrm{H}), 2.20-1.98(\mathrm{~m}, 3 \mathrm{H}), 1.91-1.80(\mathrm{~m}, 1 \mathrm{H}) ;{ }^{13} \mathrm{C}$ NMR $\left(101 \mathrm{MHz}, \mathrm{CDCl}_{3}\right) \delta 176.0,145.1,128.9,127.0,126.7,68.4,47.4$, 36.9, 33.8, 30.5; LRMS (CI) $\mathrm{m} / \mathrm{z}$ 191.1 [M+H] $]^{+}$; HRMS (FTMS-NSI) calculated for $\mathrm{C}_{12} \mathrm{H}_{15} \mathrm{O}_{2}[\mathrm{M}+\mathrm{H}]^{+}$191.1067, found 191.1064; IR $(\mathrm{ATR}) / \mathrm{cm}^{-1} 2960,2943,1740$.

\subsection{7-Methyloxepan-2-one $(13)^{53}$}

Lactone 13 was prepared as a 7:1 mixture of regioisomers, according to General Procedure 1 using 2-methylcyclohexanone 12 (0.49 $\mathrm{mL}, 4.0 \mathrm{mmol})$. The title compound was isolated after column chromatography as a colourless oil $(468 \mathrm{mg}, 3.7 \mathrm{mmol}, 91 \%, 15: 1$ mixture of regioisomers). ${ }^{1} \mathrm{H}$ NMR (400 $\mathrm{MHz}, \mathrm{CDCl}_{3}$ ) $\delta 4.43$ (app dq, $J=8.3,6.4 \mathrm{~Hz}, 1 \mathrm{H}$ major), 4.31-4.15 (m, 2H minor), $2.72-2.52$ 
(m, 2H major, $1 \mathrm{H}$ minor), 1.99-1.83 (m, 3H major, 2H minor), 1.72-1.51 (m, 3H major, 4H minor), 1.34 (d, $J=6.4 \mathrm{~Hz}, 3 \mathrm{H} \mathrm{major),}$ $1.18\left(\mathrm{~d}, J=6.7 \mathrm{~Hz}, 3 \mathrm{H}\right.$ minor); ${ }^{13} \mathrm{C} \mathrm{NMR}\left(101 \mathrm{MHz}, \mathrm{CDCl}_{3}\right.$, major isomer) $\delta 175.7,76.9,36.4,35.1,28.4,23.0,22.7$; LRMS (CI) $m / z$ $129.0[\mathrm{M}+\mathrm{H}]^{+} ;$IR (ATR) $/ \mathrm{cm}^{-1} 2987,2901,1716$.

4.8. 7-Ethyloxepan-2-one $(15)^{57}$

Lactone $\mathbf{1 5}$ was prepared as a 15:1 mixture of regioisomers, according to General Procedure 1 using 2-ethylcyclohexanone 14 (0.56 $\mathrm{mL}, 4.0 \mathrm{mmol})$. The title compound was isolated after column chromatography as a colourless oil $(410 \mathrm{mg}, 2.9 \mathrm{mmol}, 72 \%, 12: 1$ mixture of regioisomers). ${ }^{1} \mathrm{H}$ NMR $\left(400 \mathrm{MHz}, \mathrm{CDCl}_{3}\right) \delta 4.26-4.24(\mathrm{~m}, 2 \mathrm{H}$ minor), 4.16 (t app d, $J=7.8,5.3 \mathrm{~Hz}, 1 \mathrm{H}$ major), $2.71-2.54$ (m, 2H major), 2.49-2.39 (m, 1H minor) , 2.03-1.84 (m, 3H major, 2H minor), 1.80-1.67 (m, 1H major, 2H minor), 1.66-1.51 (m, 4H major, $2 \mathrm{H}$ minor), $1.47-1.37$ (m, $2 \mathrm{H}$ minor) 0.98 (t, $J=7.4 \mathrm{~Hz}, 3 \mathrm{H}$ major), 0.95 (t, $J=7.6 \mathrm{~Hz}, 3 \mathrm{H}$ minor); ${ }^{13} \mathrm{C} \mathrm{NMR}\left(101 \mathrm{MHz}, \mathrm{CDCl}_{3}\right.$, major isomer) $\delta 175.9,81.9,35.1,34.3,29.5,28.5,23.2,10.0$; LRMS (CI) $m / z$ 143.1 [M+H] ${ }^{+}$; HRMS (TOF-EI) calculated for $\mathrm{C}_{8} \mathrm{H}_{14} \mathrm{O}_{2}$ $[\mathrm{M}+\bullet]$ 142.0994, found 142.0997; IR (ATR)/ $\mathrm{cm}^{-1} 2970,2931,1721$.

4.9. 7-Phenyloxepan-2-one $(17)^{58}$

Lactone 17 was prepared as a 20:1 mixture of regioisomers, according to General Procedure 1 using 2-phenylcyclohexanone 16 (697 $\mathrm{mg}, 4.0 \mathrm{mmol})$. The title compound was isolated after column chromatography as a white solid (683 $\mathrm{mg}, 3.6 \mathrm{mmol}, 90 \%, 20: 1 \mathrm{mixture}$ of regioisomers). m.p. $68-69^{\circ} \mathrm{C}$ (lit. $\left.67-68{ }^{\circ} \mathrm{C}\right) ;{ }^{58}{ }^{1} \mathrm{H} \mathrm{NMR}\left(400 \mathrm{MHz}, \mathrm{CDCl}_{3}\right) \delta 7.60-7.21(\mathrm{~m}, 5 \mathrm{H}$ major, $5 \mathrm{H}$ minor), 5.29 (app d, $J=$ $9.4 \mathrm{~Hz}, 1 \mathrm{H}$ major), 4.40-4.35 (m, 2H minor), 3.91-3.86 (m, 1H minor), 2.77 (dd, $J=7.7,3.7 \mathrm{~Hz}, 2 \mathrm{H}$ major), $2.18-1.97$ (m, 4H major, 4H minor), 1.84-1.64 (m, 2H major, 2H minor); ${ }^{13} \mathrm{C} \mathrm{NMR}\left(101 \mathrm{MHz}, \mathrm{CDCl}_{3}\right) \delta 175.0,140.9,128.7,128.3,126.0,82.3,37.6,35.1$, 28.8, 23.0; LRMS (CI) $\mathrm{m} / z$ 191.1 [M+H] $]^{+}$; HRMS (FTMS-NSI) calculated for $\mathrm{C}_{12} \mathrm{H}_{15} \mathrm{O}_{2}[\mathrm{M}+\mathrm{H}]^{+}$191.1067, found 191.1063; IR (ATR)/cm $\mathrm{cm}^{-1} 2928,2868,1717$.

\subsection{1,4,8-Trioxaspiro[4.6]undecan-9-one (19)}

Lactone 19 was prepared according to General Procedure 1 using 1,4-dioxaspiro[4.5]decan-8-one 18 (625 mg, $4.0 \mathrm{mmol})$. The title compound was isolated as a colourless oil (244 mg, $1.4 \mathrm{mmol}, 36 \%) .{ }^{1} \mathrm{H}$ NMR (400 MHz, $\left.\mathrm{CDCl}_{3}\right) \delta 4.28-4.19(\mathrm{~m}, 2 \mathrm{H}), 3.96-3.91(\mathrm{~m}$, 4H), 2.70-2.60 (m, 2H), 2.00-1.92 (m, 2H), 1.91-1.81 (m, 2H); ${ }^{13} \mathrm{C}$ NMR $\left(101 \mathrm{MHz}, \mathrm{CDCl}_{3}\right) \delta$ 175.5, 107.8, 64.8, 64.3, 39.0, 32.7, 28.8; LRMS (CI) $\mathrm{m} / z$, $173.1[\mathrm{M}+\mathrm{H}]^{+}$; HRMS (FTMS-NSI) calculated for $\mathrm{C}_{8} \mathrm{H}_{13} \mathrm{O}_{4}[\mathrm{M}+\mathrm{H}]^{+} 173.0808$, found 173.0804; IR (ATR)/cm ${ }^{-1}$ 2970, 2889, 1729.

\subsection{1. (4R,7S)-7-Isopropyl-4-methyloxepan-2-one $(21)^{59}$}

Lactone 21 was prepared according to General Procedure 1 using (-)-menthone 20 (0.69 mL, 4.0 mmol). The title compound was $\begin{array}{lllllllll}\text { isolated as a } & \text { colourless } & \text { oil } & (344 & \mathrm{mg}, & 2.0 & \mathrm{mmol},\end{array}$ $[\alpha]_{\mathrm{D}}^{20}-20.6\left(\mathrm{c} 0.72, \mathrm{CHCl}_{3}\right)$, lit. $-19.7 ;{ }^{59}{ }^{1} \mathrm{H}$ NMR $\left(400 \mathrm{MHz}, \mathrm{CDCl}_{3}\right) \delta 4.03(\mathrm{app} \mathrm{dd}, J=9.2,4.5 \mathrm{~Hz}, 1 \mathrm{H}), 2.65-2.39(\mathrm{~m}, 2 \mathrm{H}), 2.03-$ $1.77(\mathrm{~m}, 4 \mathrm{H}), 1.66-1.52(\mathrm{~m}, 1 \mathrm{H}), 1.35-1.21(\mathrm{~m}, 1 \mathrm{H}), 1.03(\mathrm{~d}, J=6.7 \mathrm{~Hz}, 3 \mathrm{H}), 0.97(\mathrm{~d}, J=6.8 \mathrm{~Hz}, 3 \mathrm{H}), 0.96(\mathrm{~d}, J=6.8 \mathrm{~Hz}, 3 \mathrm{H}) ;{ }^{13} \mathrm{C}$ NMR $\left(101 \mathrm{MHz}, \mathrm{CDCl}_{3}\right) \delta 175.1,84.9,42.7,37.6,33.5,31.1,30.5,24.1,18.5,17.3 ; \mathrm{LRMS}(\mathrm{CI}) \mathrm{m} / z$ 171.1 [M+H] ${ }^{+}$; HRMS (FTMS-NSI) calculated for $\mathrm{C}_{10} \mathrm{H}_{19} \mathrm{O}_{2}[\mathrm{M}+\mathrm{H}]^{+}$171.1380, found 171.1376; IR (ATR)/cm ${ }^{-1} 2970,2928,1722$.

\subsection{Tetrahydro-2H-pyran-2-one $(23)^{60}$}

Lactone 23 was prepared according to General Procedure 1 using cyclopentanone $22(0.36 \mathrm{~mL}, 4.0 \mathrm{mmol})$. The title compound was isolated as a colourless oil $(118 \mathrm{mg}, 1.1 \mathrm{mmol}, 30 \%)$. ${ }^{1} \mathrm{H}$ NMR $\left(400 \mathrm{MHz}, \mathrm{CDCl}_{3}\right) \delta 4.38-4.29(\mathrm{~m}, 2 \mathrm{H}), 2.54(\mathrm{t}, J=7.1 \mathrm{~Hz}, 2 \mathrm{H}), 1.97-$ $1.78(\mathrm{~m}, 4 \mathrm{H}) ;{ }^{13} \mathrm{C}$ NMR $\left(101 \mathrm{MHz}, \mathrm{CDCl}_{3}\right) \delta 171.5,69.5,29.9,22.4,19.2 ; \mathrm{LRMS}(\mathrm{CI}) \mathrm{m} / \mathrm{z} 104.0[\mathrm{M}+\mathrm{H}]^{+} ; \mathrm{IR}(\mathrm{ATR}) / \mathrm{cm}^{-1} 2970,2934$, 1724.

4.13. Dihydrofuran-2(3H)-one $(25)^{60}$

Lactone 25 was prepared according to General Procedure 1 using cyclobutanone $\mathbf{2 4}(0.30 \mathrm{~mL}, 4.0 \mathrm{mmol})$. The title compound was isolated as a colourless oil $(292 \mathrm{mg}, 3.4 \mathrm{mmol}, 85 \%) .{ }^{1} \mathrm{H}$ NMR $\left(400 \mathrm{MHz}, \mathrm{CDCl}_{3}\right) \delta 4.33(\mathrm{t}, J=7.0 \mathrm{~Hz}, 2 \mathrm{H}), 2.57-2.44(\mathrm{~m}, 2 \mathrm{H}), 2.35-$

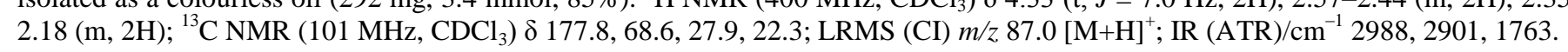

4.14. Rel-(1R,3R,6S,8S)-4-Oxatricyclo[4.3.1.1 $\left.1^{3,8}\right]$ undecan-5-one $(27)^{61}$

Lactone 27 was prepared according to General Procedure 1 using 2-adamantanone 26 (600 mg, 4.0 mmol). The title compound was isolated as a white solid $(545 \mathrm{mg}, 3.3 \mathrm{mmol}, 82 \%)$. m.p. $>250{ }^{\circ} \mathrm{C}\left(\mathrm{lit} .288-290{ }^{\circ} \mathrm{C}\right) ;{ }^{61}{ }^{1} \mathrm{H} \mathrm{NMR}\left(400 \mathrm{MHz}, \mathrm{CDCl}_{3}\right) \delta 4.51-4.43(\mathrm{~m}, 1 \mathrm{H})$, $3.06(\operatorname{app~t}, J=5.8 \mathrm{~Hz}, 1 \mathrm{H}), 2.14-1.88(\mathrm{~m}, 8 \mathrm{H}), 1.86-1.78(\mathrm{~m}, 2 \mathrm{H}), 1.77-1.70(\mathrm{~m}, 2 \mathrm{H}) ;{ }^{13} \mathrm{C} \mathrm{NMR}\left(101 \mathrm{MHz}, \mathrm{CDCl}_{3}\right) \delta 179.1,73.3$, 41.4, 35.9, 33.9, 31.1, 26.0; LRMS (CI) m/z 167.0 [M+H] ${ }^{+}$; HRMS (FTMS-NSI) calculated for $\mathrm{C}_{10} \mathrm{H}_{15} \mathrm{O}_{2}[\mathrm{M}+\mathrm{H}]^{+} 167.1067$, found 167.1063; IR (ATR)/(cm ${ }^{-1} 2988,2911,1717$.

\section{Acknowledgements}

The authors thank the EPSRC and University of Strathclyde for financial support and the EPSRC Mass Spectrometry Service, Swansea for high-resolution spectra.

\section{References and notes}

1. Baeyer, A.; Villiger, V. Ber. Dtsch. Chem. Ges. 1899, 32, 3625-3627.

2. Li, J. Name Reactions; Springer: Berlin, Heidelberg, 2009, pp. 10-12.

3. Brink, G. J.; Arends, I. W. C. E.; Sheldon, R. A. Chem. Rev. 2004, 104, 4105-4123.

4. Renz, M.; Meunier, B. Eur. J. Org. Chem. 1999, 1999, 737-739. 
5. Heaney, H. Aldrichimica Acta 1993, 26, 35-45.

6. Cooper, M. S.; Heaney, H.; Newbold, A. J.; Sanderson, W. R. Synlett 1990, 533-535.

7. McKillop, A.; Sanderson, W. R. Tetrahedron 1995, 51, 6145-6166.

8. Muzart, J. Synthesis 1995, 47, 1325-1347.

9. McKillop, A.; Sanderson, W. R. J. Chem. Soc. Perkin Trans. 1 2000, 471-476.

10. Corma, A.; Nemeth, L. T.; Renz, M.; Valencia, S. Nature 2001, 412, 423-425.

11. Luo, H. Y.; Bui, L.; Gunther, W. R.; Min, E.; Román-Leshov, Y. ACS Catal. 2012, 2, 2695-2699.

12. Hara, T.; Hatakeyama, M.; Kim, A.; Ichikuni, N.; Shimazu, S. Green Chem. 2012, 14, 771-777.

13. Kirumakki, S.; Samarajeewa, S.; Harwell, R.; Mukherjee, A.; Herber, R. H.; Clearfield, A. Chem. Commun. 2008, 5556-5558.

14. Lei, Z.; Zhang, Q.; Wang, R.; Ma, G.; Jia, C. J. Organomet. Chem. 2006, 691, 5767-5773.

15. Corma, A.; Iborra, S.; Mifsud, M.; Renz, M.; Susarte, M. Adv. Synth. Catal. 2004, 346, 257-262.

16. Hao, X.; Yamazaki, O.; Yoshida, A.; Nishikido, J. Green Chem. 2003, 5, 524-528.

17. Renz, M.; Blasco, T.; Corma, A.; Fornés, V.; Jensen, R.; Nemeth, L. Chem. Eur. J. 2002, 8, $4708-4717$.

18. Zhang, X.; Ye, J.; Yu, L.; Shi, X.; Zhang, M.; Xu, Q.; Lautens, M. Adv. Synth. Catal. 2015, 357, 955-960.

19. Martins, L. M. D. R. S.; Alegria, E. C. B. A.; Smoleński, P.; Kuznetsov, M. L.; Pombeiro, A. J. L. Inorg. Chem. 2013 , 52 , $4534-4546$.

20. Uyanik, M.; Nakashima, D.; Ishihara, K. Angew. Chem. Int. Ed. 2012, 51, 9093-9096.

21. Cavarzan, A.; Bianchini, G.; Sgarbossa, P.; Lefort, L.; Gladiali, S.; Scarso, A.; Strukul, G. Chem. Eur. J. 2009, 15, 7930-7939.

22. Conte, V.; Floris, B.; Galloni, P.; Mirruzzo, V.; Scarso, A.; Sordi, D.; Strukul, G Green Chem. 2005, 7, $262-266$.

23. Michelin, R. A.; Pizzo, E.; Scarso, A.; Sgarbossa, P.; Strukul, G.; Tassan, A. Organometallics 2005, $24,1012-1017$.

24. Malkov, A. V.; Friscourt, F.; Bell, M.; Swarbrick, M. E.; Kočovský, P. J. Org. Chem. 2008, 73, $3996-4003$.

25. Yoshida, A.; Yoshimura, M.; Uehara, K.; Hikichi, S.; Mizuno, N. Angew. Chem. Int. Ed. 2006, 45, $1956-1960$.

26. Watanabe, A.; Uchida, T.; Irie, R.; Katsuki, T. Proc. Natl. Acad. Sci. USA 2004, 101, 5737-5742.

27. Flourat, A. L.; Peru, A. A. M.; Teixeira, A. R. S.; Brunissen, F.; Allais, F. Green Chem. 2015, 17, 404-412.

28. Kotlewska, A. J.; van Rantwijk, F.; Sheldon, R. A.; Arends, I. W. C. E. Green Chem. 2011, 13, 2154-2160.

29. Peris, G.; Miller, S. J. Org. Lett. 2008, 10, 3049-3052.

30. Xu, S.; Wang, Z.; Li, Y.; Zhang, X.; Wang, H.; Ding, K. Chem. Eur. J. 2010, 16, 3021-3035.

31. Xu, S.; Wang, Z.; Zhang, X.; Zhang, X.; Ding, K. Angew. Chem. Int. Ed. 2008, 47, 2840-2843.

32. Murahashi, S.-I.; Ono, S.; Imada, Y. Angew. Chem. Int. Ed. 2002, 41, 2366-2368.

33. Payne, G. B.; Williams, P. H. J. Org. Chem. 1961, 26, 651-659.

34. Payne, G. B.; Deming, P. H.; Williams, P. H. J. Org. Chem. 1961, 26, 659-661.

35. Okazaki, H.; Hanaya, K.; Shoji, M.; Hada, N.; Sugai, T. Tetrahedron 2013, 69, 7931-7935.

36. Frank, W. C. Tetrahedron: Asymmetry 1998, 9, 3745-3749.

37. Bachmann, C.; Gesson, J.-P.; Renoux, B.; Tranoy, I. Tetrahedron Lett. 1998, 39, 379-382.

38. Frank, W. C. Tetrahedron: Asymmetry 1998, 9, 3745-3749.

39. Bachmann, C.; Gesson, J.-P.; Renoux, B.; Tranoy, I. Tetrahedron Lett. 1998, 39, 379-382.

40. Wong, O. A.; Shi, Y. Chem. Rev. 2008, 108, 3958-3987.

41. Burke, C. P.; Shu, L.; Shi, Y. J. Org. Chem. 2007, 72, 6320-6323.

42. Della Salla, G.; Lattanzi, A. ACS Catal. 2014, 4, 1234-1245.

43. Uraguchi, D.; Tsutsumi, R.; Ooi, T. J. Am. Chem. Soc. 2013, 135, 8161-8164.

44. Radziszewski, B. Ber. Dtsch. Chem. Ges. 1885, 18, 355-356.

45. Vacque, V.; Dupuy, N.; Sombret, B.; Huvenne, J. P.; Legrand, P. J. Mol. Struct. 1996, 384, $165-174$.

46. Ji, L.; Wang, Y.-N.; Qian, C.; Chen, X. Z. Synth. Commun. 2013, 43, 2256-2264.

47. Llamas, R.; Jiménez-Sanchidrián, C.; Ruiz, J. R. Appl. Catal., B 2007, 72, 18-25.

48. Llamas, R.; Jiménez-Sanchidrián, C.; Ruiz, J. R. Tetrahedron 2007, 63, 1435-1439.

49. For a review on surface-based catalysts used in the Baeyer-Villiger reaction, please refer to Jiménez-Sanchidrián, C.; Ruiz, J. R. Tetrahedron 2008, 64, 2011-2026.

50. Dragan, A.; Kubczyk, T. M.; Rowley, J. H.; Sproules, S.; Tomkinson. N. C. O. Org. Lett. 2015, 17, $2618-2621$.

51. Picon, S.; Rawling, M.; Campbell, M.; Tomkinson, N. C. O. Org. Lett. 2012, 14, 6250-6253.

52. Neimann, K.; Neumann, R. Org. Lett. 2000, 2, 2861-2863.

53. Marell, D. J.; Emond, S. J.; Kulshrestha, A.; Hoye, T. R. J. Org. Chem. 2014, 79, 752-758.

54. Shioji, K.; Matsuo, A.; Okuma, K.; Nakamura, K.; Ohno, A. Tetrahedron Lett. 2000, 41, 8799-8802.

55. Rothenberg, G.; Downie, A. P.; Raston, C. L.; Scott, J. L. J. Am. Chem. Soc. 2001, 123, 8701-8708.

56. Yato, M.; Homma, K.; Ishida, A. Tetrahedron 2001, 57, 5353-5359.

57. van Buijtenen, J; van As, B. A. C.; Meuldijk, J.; Palmans, A. R. A.; Vekemans, J. A. J. M.; Hulshof, L. A.; Meijer, E. W. Chem. Commun 2006, 31693171.

58. Zhang, B.; Han, L.; Li, T.; Yan, J.; Yang, Z. Synth. Commun. 2014, 44, 1608-1613.

59. Fink, M. J.; Fischer, T. C.; Rudroff, F.; Dudek, H.; Fraaije, M. W.; Mihovilovic, M. D. J. Mol. Catal. B: Enzym. 2011, 73, 9-16.

60. Xie, X.; Stahl, S. S. J. Am. Chem. Soc. 2015, 137, 3767-3770.

61. Banister, S. D.; Yoo, D. T.; Chua, S. W.; Cui, J.; Mach, R. H.; Kassiou, M. Bioorg. Med. Chem. Lett. $2011,21,5289-5292$. 